\title{
Spectral impact on multi-junction solar cells obtained by means of component cells of a different technology
}

\author{
Ruben Núñez*, Marta Victoria, Stephen Askins, Ignacio Antón, César Domínguez, Rebeca Herrero, \\ Gabriel Sala
}

Instituto de Energía Solar, Universidad Politécnica de Madrid, Madrid, Spain

*ruben.nunez@ies.upm.es

\begin{abstract}
Spectral indices can be defined to characterize how the solar spectrum affects photovoltaic devices. The use of such indices is particularly important in technologies which are highly dependent on spectral variations, such as tandem, or multi-junction (MJ), solar cells. In this paper, we analyze the capability of three effective irradiance values, given by the photocurrents of the components of triple-junction solar cells, and covering different spectral bands, to define the spectrum condition. Even though these parameters will be based on a particular type of MJ solar cell, they provide enough information to accurately assess the spectral impact on other types of MJ solar cell technologies with significantly different spectral responses. Consequently, specific component cells for each MJ solar cell technology may not be necessary needed and a reference technology could be used for all them, providing a way for the future standardization of these devices as a mean of defining the solar spectral condition.
\end{abstract}

1. Introduction

Spectral indices are used to describe the dependence of solar radiation on wavelength. From a theoretical point of view, an ideal set of spectral indices should allow bijective (unambiguous) identification of a spectrum through a unique set of values. In other words, a particular set of spectral indices corresponds to a solar spectrum, but only one, and the reciprocal is also true, a particular solar spectrum corresponds to one, and only one, set of spectral indices.

In photovoltaic (PV) technology, spectral indices are defined trying to reflect how changes in the spectrum affect the performance of a solar cell. This is particularly relevant for systems where multijunction (MJ) solar cells are involved due to their large spectral sensitivity. One of the main objectives of defining a spectral index for PV or concentrator PV (CPV) is to account for spectral mismatch, i.e., understand and quantify the effect on the cell performance of a particular spectral change. In a real-world scenario, a practical spectral index would have a certain tolerance that is unavoidable and must be quantified. In practice this means that there is a set of spectra that produce the same spectral index.

Spectral variations in the solar resource are almost entirely caused by earth's atmosphere. The sun's elevation and the altitude of the site, both characterized by the air mass (AM), determine the optical length that sunlight must pass through before reaching the Earth's surface. Particles and molecules contained in the atmosphere, mostly aerosols (given by the aerosol optical depth, AOD) and precipitable water (PW), determine how radiation at different wavelengths is absorbed by a given air mass. Combined, these factors modify the spectral distribution that finally reaches the PV system.

Consequently, the three most important atmospheric factors that have impact on solar spectrum and affect PV performance are AM, AOD, and PW. Although there are other atmospheric components that affect solar spectrum, they are usually neglected because of their low influence on PV devices. Hence, if those values are known at a particular moment, the spectrum can be essentially described. Therefore, the tuple (AM, AOD, PW) could be regarded as a spectral measure. However, these three parameters do not allow for straightforward assessment of spectral variations, since their influence on spectrum is intricate, unbalanced between the three and can only be evaluated through complex atmospheric models [1]-[3]. In this regard, additionally to the proposed work, some analytical straight models have been proposed [4][6].

Throughout this paper, we will consider the spectral uniqueness of spectral indices in terms of how the spectrum affects the performance of a particular PV technology. In that regard, several indices have been proposed to evaluate spectral mismatch in silicon and MJ solar cells, i.e., the differences in the photocurrents of the device provided by impinging light of the same power but different spectral content. A detailed review of several spectral indices can be found in [7]. Some spectral indices can be directly measured using the photocurrent generated by a sensor (usually a photovoltaic solar cell) while others are indirectly estimated by integrating 
spectral irradiance weighted by a certain device spectral response.

Spectral indices are particularly significant for those PV technologies highly influenced by spectral variations, such as tandem, or MJ solar cells. The CPV community, where MJ solar cells are widely used, has devoted significant efforts to create spectral indices such as Z parameters [8] and Spectral Matching Ratios (SMRs) [9]-[11] capable of defining and constraining the solar spectral condition in terms relevant to MJ solar cells performance. One of the main advantages of these indices is that they can be measured with simple sensors, based on isotypes or component cells, installed into an instrument similar to a pyrheliometer. In addition, they can be used not only outdoors but also indoors since its fast response is compatible with flash lamps used in solar simulators. These indices are device-dependent (because they are based on the relationships among the photocurrents generated by the subcells within the MJ device), so they can also be used to assess the influence of the spectrum variation on MJ solar cells. SMR indices have been included in the standard for power rating of CPV (IEC-62670-3) as a mean of defining a prevailing spectral conditions similar to the reference spectrum [12] for both indoor and outdoor power rating of CPV modules.

Unlike conventional PV technology based on silicon single junction solar cells, MJ solar cells based on III$\mathrm{V}$ semiconductors are constantly evolving, introducing new materials with different band gaps and spectral responses, or even a higher number of subcells (from the current standard of three junctions, we expect to pass to 4 or 5 junctions in coming years.) Since SMR indices are device dependent, a key question is how important it is to match the sensor to the device under test. For instance, if prevailing conditions are fixed by SMR indices of a first MJ technology (which we will call primary), what is the uncertainty associated (or equivalent tolerance) with the measurement if the device under test uses a secondary MJ technology with different spectral response, bandgaps and perhaps number of junctions? Could the prevailing conditions of the secondary technology be deduced from the primary? Should prevailing conditions always be fixed by using isotype cells of the same technology of the device under test? Or could a reference technology be standardized and used for other MJ cells?

The answers to all those questions depend the on capacity of the spectral indices, in this case SMRs of a primary technology, to identify a spectrum that is unique (considering a given tolerance) with respect to its influence on the performance of the solar cells. If that were the case, such indices could be used for any MJ technology. This does not necessary mean that a set of values of SMR indices will indicate the same prevailing conditions for both primary and secondary technologies. But it ensures at least that mathematical relationships for the SMRs of a secondary technology (as a function of the primary SMRs) should exist and, consequently, the secondary prevailing conditions could be inferred. Since all solar spectra can be generated by AM, AOD, and PW spectral parameters, it seems sensible as a practical matter to simplify the mapping from the spectral domain to the technology response codomain using an SMR approach.

In this paper, we analyze the capability of the SMRs of a primary technology to define the solar spectra for PV applications using MJ solar cells of a different secondary technology and then estimating the corresponding error or allowed tolerance. We have focused on state-of-the-art triple junction (3J) and four junction (4J) technologies which are currently in the market for CPV applications. For every solar cell technology under analysis, first, the spectral indices are directly calculated for a synthetic spectra data set using the spectral response of that particular technology. Then, they are compared to the spectral indices derived from a primary technology with different spectral response. In order to complement the results, and only for one of the technologies under analysis, spectral indices experimentally measured for several days are also compared.

The article is organized as follows. Section 2 describes the synthetic spectra data set used for the analysis. Section 3 includes the definition of the SMRs and the effective irradiances. Section 4 provides a detailed description of the methodology. Section 5 contains the results and discussion which comprises results on $3 \mathrm{~J}$ solar cells with similar spectral responses (section 5.1) and results on $3 \mathrm{~J}$ and 4J solar cells whose spectral responses show larger differences (section 5.2). Finally, conclusions are summarized in section 6 .

\section{Spectra data set}

The source of data for the analyses carried out in this paper is a set of synthetic direct spectra generated with the atmospheric modeling software SMARTS [13] throughout a period of one year and using as inputs the measured spectral parameters (AM, AOD, PW) from the AERONET network [14] (which includes 
clear-sky data and samples every 15 minutes). In order to illustrate the results, data measured in the AERONET station located in Madrid is initially shown. SMARTS has been configured based on an urban model for aerosols [13], an altitude of $695 \mathrm{~m}$ and an average air pressure of $933 \mathrm{hPa}$. The rest of SMARTS parameters, such as ozone and $\mathrm{CO}_{2}$, have negligible influence on this analysis and have been set to reference values ${ }^{1}$.

Figure 1 shows the set of synthetic spectra generated by SMARTS using AERONET information measured in the station located in Madrid throughout 2014.

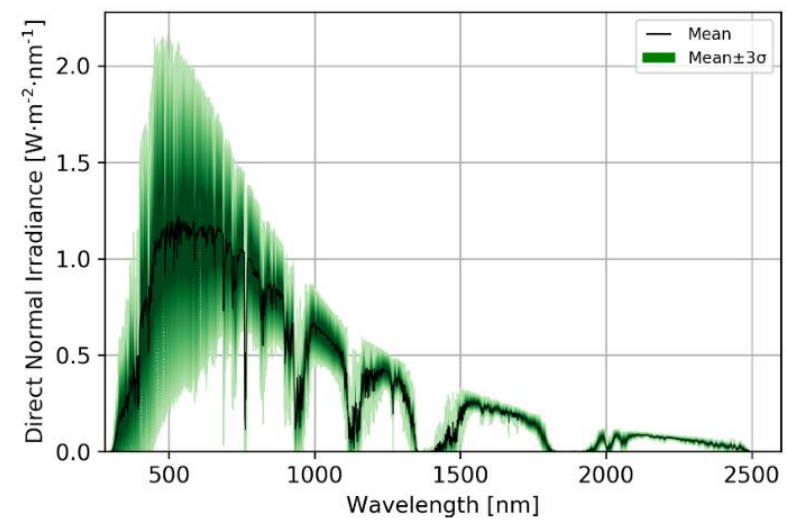

Figure 1. Synthetic spectra obtained with SMARTS and using data from the Madrid AERONET station (year 2014). For every wavelength, the black line represents the mean irradiance value and the green area shows values within the mean \pm 3 times the standard deviation.

Typically, a solar spectrum is given as an array of irradiance values per unit wavelength, within the spectral range of interest. For example, the AM1.5D G173-03 reference spectrum [15] is composed by 2002 irradiance values corresponding to wavelengths that range from 280 to $4000 \mathrm{~nm}$. Therefore, the number of different combinations that could potentially be described with that resolution is enormous, ${ }^{2}$ even if several of these arrays are no physically possible solar spectra. Nevertheless, as pointed out above, only three spectral parameters (AM, AOD, PW) substantially influence the solar spectra in the wavelength range used by MJ solar cells, suggesting that the solar spectra can be described with three independent variables. In the next section, two set of values, both derived from the photocurrents generated by the

\footnotetext{
1 As a representative example, the sensitivity analysis based on SMARTS showed that a $\pm 3 \%$ variation in the atmospheric pressure implies a change on the photocurrent of the GaAs top subcell (the most affected by air pressure in $3 \mathrm{~J}$ solar cells) lower than $0.35 \%$. This
}

subcells within a MJ solar cell, are proposed as threedimensional spectra indices.

\section{Spectral matching ratios (SMRs) and effective direct normal irradiances as spectral indices}

Since AM, AOD and PW are the physical variables responsible of changes in solar spectrum, they could be used as a set of spectral indices. However, they do not provide a direct measure of spectrum but, rather, a way of reconstructing spectra through complex atmospheric models. Therefore, they are not well suited for quantifying spectral variations. Furthermore, AOD and PW have to be estimated based on the measurements of equipment such as photometers, the SolarSIM-D2 by Spectrafy or relying on satellite data [12], [16].

SMRs are spectral indices that were initially developed for CPV technology as a significant measure of spectral distributions [9], [11], [17], [18]. The definition of SMR is technology dependent as it is influenced by the bandgaps of the subcells composing the MJ stack. In the following section we will explore whether the application of the SMRs derived from a particular MJ technology can be generalized to other types of cells.

SMR quantifies the difference between a particular irradiance spectrum and another reference spectrum, e.g., AM1.5D described in ASTM G173-03 [15]. SMR is defined by dividing the ratio between the currents photogenerated by two subcells under the spectrum in study by the same ratio under the reference spectrum AM1.5D [9]-[11],

$$
\begin{aligned}
& S M R_{\text {subcell }_{i}}^{\text {subcell }_{i}}=\frac{I_{\text {L,ratio }_{\text {subcell }}} \text { subcell }_{i}}{\left.I_{L, \text { ratio }_{\text {subcell }_{j}}}\right]_{\text {ref }}} \\
& =\frac{\frac{I_{L, \text { subcell }_{i}}}{I_{L, \text { subcell }_{j}}}}{I_{L, \text { subcell }_{\text {ref }}}}
\end{aligned}
$$

where $I_{L, \text { subcell }}$ represents the photocurrent of subcell $i$ when illuminated with a particular spectral irradiance distribution (spectrum in study) and

variation represents well Madrid where $99 \%$ of the time air pressure is within $\pm 2.1 \%$ of the reference value. Other parameters showed even lower influence.

${ }^{2}$ Considering 2002 values per spectrum and a resolution of eight bits per value, there are $256^{2002}$ combinations. 
$I_{L, \text { subcell }} i_{\text {ref }}$ stands for the photocurrent of subcell $i$ under the reference spectrum.

For a particular MJ solar cell technology, an effective direct normal irradiance $B_{\text {subcell } i}$ is defined for every subcell $i$ as the ratio of its photocurrent under a particular spectral distribution to that under the reference spectrum AM1.5D [19], multiplied by the reference $\mathrm{DNI}$, that is,

$$
B_{\text {subcell }_{i}}=\frac{I_{L, \text { subcell }_{i}}}{I_{L, \text { subcell }_{\text {ref }}}} B_{\text {ref }}
$$

where $B_{\text {ref }}$ is the direct normal irradiance (DNI) at which the calibrated photocurrent of subcell $i$ under the reference spectrum, $I_{L, \text { subcell }_{i} \text { rf }}$, is obtained. In the case of $3 \mathrm{~J}$ solar cells, a set of effective irradiance values for the top, middle and bottom subcells can be defined. Using this definition, two spectra that produce the same photocurrent in a particular subcell (and thus the same effective irradiance) can be considered "equivalent" for that particular subcell. Hence, SMR can also be defined using the effective irradiance $B_{\text {subcell }_{i}}$ :

$$
S M R_{\text {subcell }_{j}}^{\text {subcell }_{i}}=\frac{B_{\text {subcell }_{i}}}{B_{\text {subcell }_{j}}}
$$

For $3 \mathrm{~J}$ solar cells, the three effective irradiance values $\left(B_{\text {top }}, B_{\text {mid, }}, B_{b o t}\right)$ are independent variables that cover three distinct spectral ranges of the solar spectra so they constitute a three-parameter spectral index that could potentially be used to assess the spectral impacts not only for that particular MJ technology but also for other MJ solar cell of different type.

The main advantage of effective irradiances and SMRs is that they can be measured directly using component (or isotype) solar cells. An instrument known as spectroheliometer [11] has been constructed for this purpose. Conventional spectroradiometers can also be used. In that case, the measured spectrum must be multiplied by the spectral response of each subcell and integrated over wavelengths in order to estimate the subcells photocurrents. More simply, the spectroheliometer provides a direct measurement of the three effective irradiance values $\left(B_{\text {top }}, B_{\text {mid }}, B_{\text {bot }}\right)$ from which SMRs are calculated using Equation (3).

In the case of using SMRs, three of them can be defined $\left(\mathrm{SMR}_{\text {mid }}^{\text {top }}, \mathrm{SMR}_{\text {bot }}^{\text {mid }}, \mathrm{SMR}_{\text {bot }}^{\text {top }}\right.$ ) although only two are independent. The first two are preferred by the authors of this paper because they use consecutive spectral bands. For LM-Ge component cells, the influence of $A O D$ and $A M$ affect mainly to the bands covered by the top and middle subcell while PW affects primarily to the band covered by the bottom subcell [3], [4]. Therefore these two SMRs can be easily related to the atmosphere content, AOD with $S M R_{\text {mid }}^{\text {top }}$ and PW with $\mathrm{SMR}_{\text {bot }}^{\mathrm{mid}}$, while $S M R_{\text {bot }}^{\text {top }}$ would be affected by both.

As SMRs are ratios, the absolute value of the energy contained in the spectrum is lost. To avoid this, SMRs should be used in conjunction with the DNI value. Here, DNI used is to refer to the total direct normal irradiance as measured by a broadband sensor (200$4000 \mathrm{~nm}$ ), such as a pyrheliometer. Consequently, the three-dimensional spectra index (DNI, SMR mid, $S M R_{b o t}^{\text {mid }}$ ) could also potentially provide a unique definition of the solar spectra.

\section{Methodology}

Based on the fact that the effective direct normal irradiances can be measured, we propose using $B_{\text {top, }}$ $B_{\text {mid }}$ and $B_{\text {bot }}$ to estimate other spectral variables such as the effective direct normal irradiances of others $3 \mathrm{~J}$ solar cell technologies with different spectral response. We expect this method to attain low fitting errors.

This three-dimensional spectral index $\left(B_{t o p}, B_{\text {mid, }}\right.$, $\left.B_{b o t}\right)_{1}$ can be measured by means of component (or isotype) sensors of a particular 3J solar cell technology and provides the necessary data to calculate the photocurrents of the three subcells of a MJ solar cell of the same technology. Now we want to know if the tuple $\left(B_{t o p}, B_{\text {mid, }}, B_{b o t}\right)_{2}$ for a second technology can be calculated from the tuple $\left(B_{\text {top }}\right.$ $\left.B_{\text {mid, }} B_{\text {bot }}\right)_{1}$ of a reference technology. If the answer is yes, a reference technology (e.g., lattice matched GalnP/InGaAs/Ge) could be used to define the spectrum for any other 3J solar cell technology. Component cells are not off-the-self devices, but the lattice-matched Germanium-based (LM-Ge) component cells are easier to obtain than newer technologies. Therefore, the following analysis considers a conventional LM-Ge $3 \mathrm{~J}$ solar cell, two versions of the upright metamorphic $\left(\mathrm{UMM}^{\mathrm{A}}\right.$ and $U_{M M}{ }^{B}$ ) $3 \mathrm{~J}$ cells, and a four junction (4J) solar cell technology. $\mathrm{UMM}^{\mathrm{A}}$ corresponds to the upright metamorphic architecture with low Indium content, that is, $\mathrm{Ga}_{0.5} \mathrm{In}_{0.5} \mathrm{P} / \mathrm{Ga}_{0.99} \mathrm{In}_{0.01} \mathrm{As} / \mathrm{Ge}$ [20] while $\mathrm{UMM}^{\mathrm{B}}$ corresponds to the structure $\mathrm{Ga}_{0.40} \mathrm{I} \mathrm{n}_{0.60} \mathrm{P} / \mathrm{Ga}_{0.90} \mathrm{In}_{0.10} \mathrm{As} / \mathrm{Ge}$ [21]. The External Quantum Efficiency (EQE) of the solar cell under analysis can be observed in the Figure 2 and Figure 
5. The 4J technology under analysis is the wafer bonded GalnP/GaAs/GalnAsP/GalnAs solar cell [22].

A linear model is proposed where the effective direct normal irradiance for a given secondary technology is modeled upon the effective direct normal irradiances of the subcells within a given primary technology, that is, $B_{\text {subcell }} i_{-} s e c=f\left(B_{\text {top_pri, }} B_{\text {mid_pri, }}\right.$ $B_{\text {bot_pri }}$. Jaus et al. [23] solved a similar problem using fitting equations but, in their model, the effective irradiance for the modeled subcell depends only on the corresponding subcell at the primary technology.

$$
\begin{gathered}
B_{\text {top_sec }}=c_{\text {top_top }} \cdot B_{\text {top_pri }}+c_{t o p_{-} \text {mid }} \cdot B_{\text {mid_pri }}+c_{\text {top_bot }} \cdot B_{\text {bot_pri }}+k_{\text {top }} \\
B_{\text {mid_sec }}=c_{\text {mid_top }} \cdot B_{\text {top_pri }}+c_{\text {mid_mid }} \cdot B_{\text {mid_pri }}+c_{\text {mid_bot }} \cdot B_{b o t \_p r i}+k_{\text {mid }} \\
B_{\text {bot_sec }}=c_{\text {bot_top }} \cdot B_{\text {top_pri }}+c_{\text {bot_mid }} \cdot B_{\text {mid_pri }}+c_{\text {bot_bot }} \cdot B_{\text {bot_pri }}+k_{\text {bot }}
\end{gathered}
$$

where the $c$ and $k$ coefficients are the fitting parameters, the sub-indices top/mid/bot refers to the top, middle and bottom subcells, and the subindices pri/sec refers to the primary and secondary technology respectively. By generating a representative family of spectra in SMARTS, calculating effective direct normal irradiance values for all subcells of both cell technologies, we can find the coefficients $c$ and $k$ by regression. From these modeled effective direct normal irradiance values for the secondary technology, we can calculate the corresponding $S M R_{\text {mid }}^{\text {top }}$ and $S M R_{\text {bot }}^{\text {mid }}$ with Equation (3). The coefficient obtained for different technologies are reported in the annex.

The more straightforward procedure consisting in modeling the SMRs of the secondary technology as a function of the SMRs of the primary technology has been discarded. The reason is that, since in this case the number of independent parameters are lower (two SMRs as opposed to three effective direct irradiances), larger errors (as defined in the following section) are obtained.

\section{Results and discussion}

In order to evaluate every approach, we define the coefficient of variation of the root mean square error $\mathrm{CV}_{\mathrm{RMSE}}$ using eq. (7). $\mathrm{CV}_{\mathrm{RMSE}}$ is calculated by comparing the SMRs of the secondary technology directly calculated from the synthetic spectra data set $S M R_{k}$, with the SMRs of the secondary technology derived from the primary technology $S M R_{\text {pri }_{k}}$ (using the method described in section 4 and eq. (4) to (6)).

Table 1 summarizes the values calculated for $\mathrm{CV}_{\mathrm{RMSE}}$ when different technologies are assumed as primary or secondary.

$$
C V_{R M S E}=\frac{\sqrt{\frac{\sum_{k=1}^{n}\left(S M R_{k}-S M R_{p r i}\right)^{2}}{n}}}{S M R_{p r i}}
$$

Table 1. Goodness of fit for the SMRs of several MJ solar cell technologies derived from different primary technologies. LM-Ge stands for lattice-matched Germanium-based solar cell and UMM represents upright metamorphic technology. Two different types of UMA technology (A and B) were analyzed. For both $\boldsymbol{S} \boldsymbol{M} \boldsymbol{R}_{\text {mid }}^{\text {top }}$ and $\boldsymbol{S} \boldsymbol{M} \boldsymbol{R}_{\text {bot }}^{\text {mid }}$ the goodness of fit is evaluated by means of the coefficient of variation of the root-mean-square error $C V_{R M S E}$ as defined in eq. (7).

\begin{tabular}{|c|l|c|c|c|}
\cline { 3 - 5 } \multicolumn{1}{c|}{} & \multicolumn{3}{c|}{ Secondary } \\
\cline { 2 - 5 } \multicolumn{1}{c|}{$\begin{array}{c}C V_{\text {RMSE }} \\
{\left[S M R_{\text {mid }}^{\text {top }} / S M R_{\text {bot }}^{\text {mid }}\right]}\end{array}$} & $\mathrm{LM}-\mathrm{Ge}$ & $\mathrm{UMM}^{\mathrm{A}}$ & $\mathrm{UMM}$ \\
\hline \multirow{3}{*}{ Primary } & - & $0.44 \% / 0.21 \%$ & $0.34 \% / 0.50 \%$ \\
\cline { 2 - 5 } & $\mathrm{LM}-\mathrm{Ge}^{\mathrm{B}}$ & $0.46 \% / 0.26 \%$ & - & $0.24 \% / 0.37 \%$ \\
\cline { 2 - 5 } & $\mathrm{UMM}^{\mathrm{B}}$ & $0.67 \% / 1.10 \%$ & $0.45 \% / 0.66 \%$ & - \\
\cline { 2 - 5 } & & &
\end{tabular}

The main conclusion that can be derived from Table 1 is intuitive: the closer the EQEs of the two involved MJ solar cells (primary and secondary), the better the goodness of the fit between the SMRs directly calculated from the spectra data set and the SMRs modeled upon the primary technology.
Furthermore, even if the technologies with more similar EQEs show a better fit, a $\mathrm{CV}_{\text {RMSE }}$ below $1.10 \%$ is obtained for all the modeled SMRs. This result is very promising at it implies that we could calculated the SMRs related to any $3 \mathrm{~J}$ solar cell by using the component cells of a different technology. 
As mentioned in the introduction, when characterizing CPV cells, modules, and systems based on MJ solar cells, component cells of the same technology (with the same spectral response) are commonly used to evaluate the spectral distribution of the light impinging the cell or the concentrator aperture [17], [24]. However, MJ solar are constantly evolving and usually the components cells for the latest solar cell architecture are not available. This is why the standardization of this type of sensors would be of great help. As it has just been shown, the 3J solar cell under analysis can be modeled using information provided by component cells from a different 3J architecture. In other words, the component cells of the "secondary" technology are not needed, but only the components cell of the "primary" technology. This could lead to use only one kind of component cells as a universal spectral reference. Most probably, component cells of LM-Ge solar cells would be the best option due to its currently extended use and availability.

In order to get a better understanding of the proposed methodology, two particular cases are analyzed hereafter. First, the solar cell technology under analysis (secondary) has an EQE similar (but slightly different) to the component cells of the reference technology (primary). Then, the second case evaluates the situation when the spectral response of the modeled and reference solar cells shows higher differences or even comprise a different number of subcells.
5.1. Triple-junction solar cell whose spectral response is similar to available component cells

Let's start with two technologies of $3 \mathrm{~J}$ solar cells with similar spectral responses. That is the case of the LMGe and $U_{M M}^{A}$ solar cells whose EQEs are depicted in Figure 2.

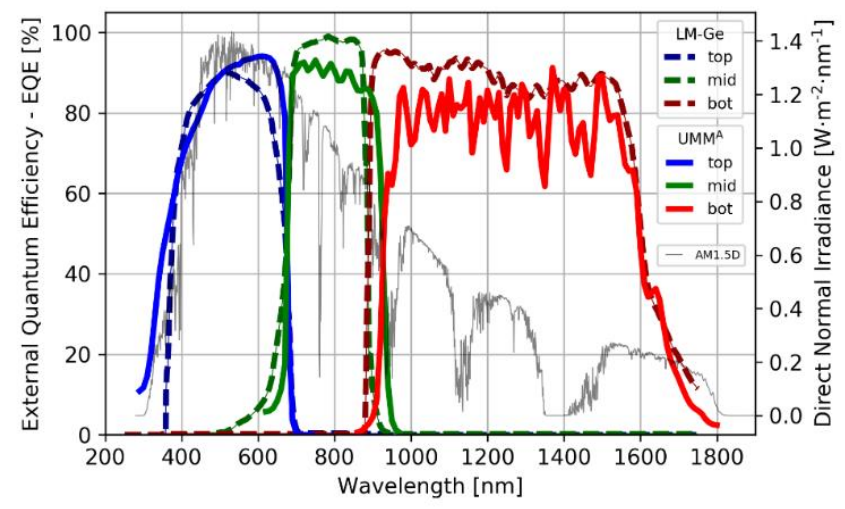

Figure 2. External Quantum Efficiencies (EQE) of the Latticematched Germanium based (LM-Ge) and Upright Metamorphic type A $\left(\mathrm{UMM}^{\mathrm{A}}\right)$ solar cell [20]. Superimposed the AM1.5D G17303 reference spectrum can be observed.

We use LM-Ge solar cell as the primary technology and the $\mathrm{UMM}^{\mathrm{A}}$ as the secondary technology.

In Figure 3, the x-axes shows the SMRs of the secondary technology directly calculated from the synthetic spectra data set. The y-axes show the SMRs of the primary technology (green dots) as well as the SMRs of the secondary technology modeled upon the primary technology following the method described in section 4 and eq. (4) to (6) (orange dots).

Upright Metamorphic A - UMM ${ }^{A}$
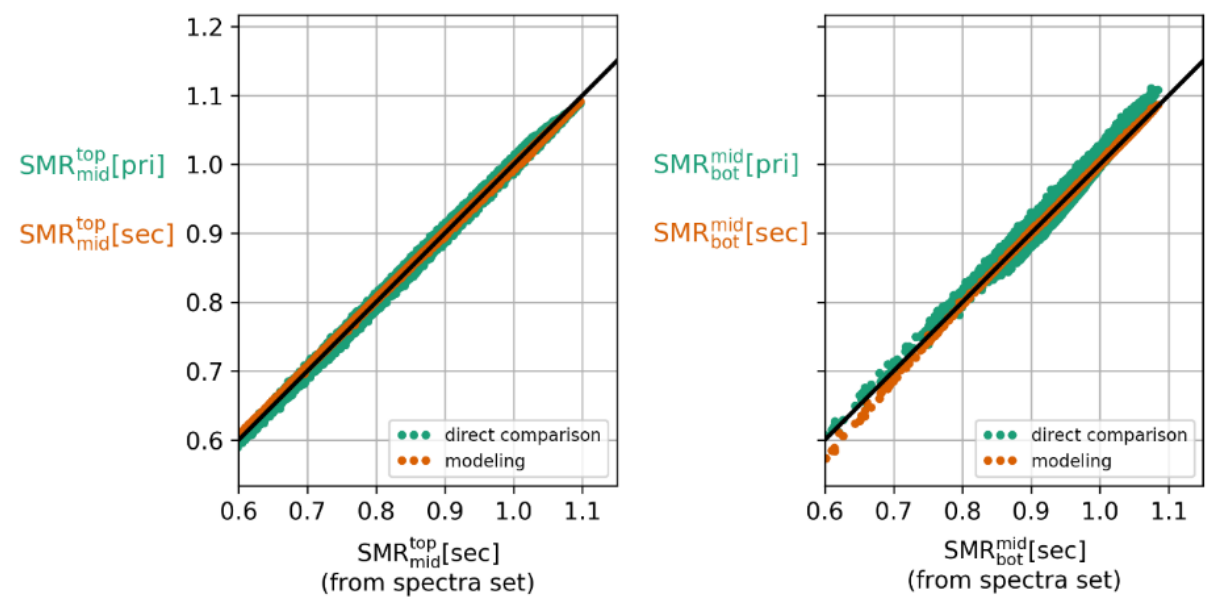

Figure 3. Correlation of $\boldsymbol{S} \boldsymbol{M} \boldsymbol{R}_{\text {mid }}^{\text {top }}$ and $\boldsymbol{S} \boldsymbol{M} \boldsymbol{R}_{\text {bot }}^{\text {mid }}$ of UMMA technology (secondary) calculated assuming LM-Ge as primary. For both graphs, $\mathrm{X}-$ axes represent the SMRs for UMM ${ }^{A}$ technology calculated from the spectra data set. The y-axes represent the SMRs for LM-Ge technology (direct comparison) as well as the SMRs for the UMMA technology derived from the effective direct normal irradiances of LM-Ge using eq. (4) to (6) (modeling). The spectral data set has been obtained by means of SMARTS and using as input information annual data (year 2014) from the Madrid AERONET station.

Looking at the direct compassion for both $\mathrm{SMR}_{\text {mid }}^{\text {top }}$ and SMR $\mathrm{bot}_{\text {bot }}^{\mathrm{mid}}$, the correlation between SMRs of LM-
Ge and $\mathrm{UMM}^{\mathrm{A}}$ (green dots in Figure 3) is fairly good. Figure 3 shows all the SMR values calculated over the 
course of a year and using data from the AERONET station in Madrid. $\mathrm{CV}_{\text {RMSE }}$ values of $0.68 \%$ and $1.14 \%$ are obtained for $\mathrm{SMR}_{\text {mid }}^{\text {top }}$ and $\mathrm{SMR}_{\text {bot }}^{\text {mid }}$ respectively. However, as shown by the orange dots in Figure 3, the correlation is even better when the method based on effective direct normal irradiances (as described in section 4) is applied. In this case, $\mathrm{CV}_{\mathrm{RMSE}}$ values decrease to $0.44 \%$ and $0.21 \%$ for $\mathrm{SMR}_{\text {mid }}^{\text {top }}$ and $\mathrm{SMR}_{\text {bot }}^{\mathrm{mid}}$ respectively. This result is also shown in Table 1. From this analysis, it can be stated that assuming SMRs values for the secondary technology identical to that of the primary technology is sufficient when the subcell gaps, and consequently the spectral bands of every subcell, are similar, as is the case of $L M-G e$ and $U_{M M}^{A}$.

A similar analysis is presented in Figure 4. In this case, real measurements were recorded with two sets of component cells of the two technologies (LMGe and $U_{M M}{ }^{A}$ ) installed in identical collimating tubes. Since the experimental campaign was carried out in Madrid during several days in the summer of 2015 , the $\mathrm{SMR}_{\text {mid }}^{\text {top }}$ achieves high values caused by the typical low AM values in this season. The $\mathrm{SMR}_{\text {bot }}^{\text {mid }}$ range is more limited because it mainly depends on local PW [25], which is typically low and constant during summer.

Upright Metamorphic A - UMM ${ }^{A}$

(experimentally measured)
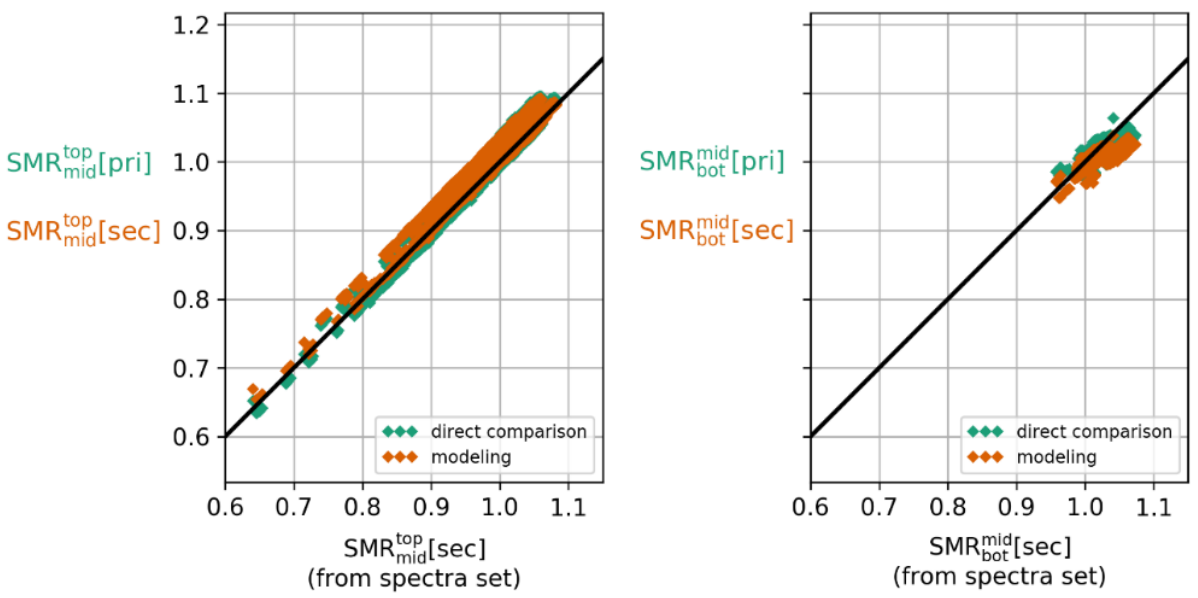

Figure 4. Correlation of $\boldsymbol{S} \boldsymbol{M} \boldsymbol{R}_{\text {mid }}^{\text {top }}$ and $\boldsymbol{S} \boldsymbol{M} \boldsymbol{R}_{\text {bot }}^{\text {mid }}$ of $U \mathrm{UM}^{\mathrm{A}}$ technology (secondary) calculated assuming LM-Ge as primary. For both graphs, $\mathrm{x}-$ axes represent the SMRs for UMMA technology experimentally measured. The y-axes represent the SMRs for LM-Ge technology experimentally measured (direct comparison) as well as the SMRs for $\mathrm{UMM}^{\mathrm{A}}$ technology derived from the effective direct normal irradiances (from LM-Ge measurements) and derived using eq. (4) to (6) (modeling). The data was obtained throughout a measurement campaign carried out in Madrid during several days in the summer of 2015 using two sets of component cells (one of LM-Ge and other of $\mathrm{UMM}^{\mathrm{A}}$ technology).

In this case, the CV RMSE for the direct comparison of the SMRs for the two technologies are $0.87 \%$ and $0.90 \%$ for $\mathrm{SMR}_{\text {mid }}^{\text {top }}$ and $\mathrm{SMR}_{\text {bot }}^{\text {mid }}$ respectively. However, when applying the model to calculate SMRs for $U_{M M}^{A}$ based on the effective direct normal irradiances measured for the LM-Ge (Eq. (4) to (6)), the $\mathrm{CV}_{\mathrm{RMSE}}$ values decreased to $0.58 \%$ and $0.65 \%$. We believe that the improvement is more significative in the experimental case due to the restricted spectral variations found during the measurements campaign.

\subsection{Multi-junction solar cells whose spectral response is fairly different to available component cells}

In this subsection, the analysis is performed for a pair of technologies with more uneven spectral responses. This is the case presented in Figure 5 left, where the EQE of the middle subcell has been expanded in the $\mathrm{UMM}^{\mathrm{B}}$ architecture reducing the spectral range of the bottom. The most relevant consequence is that the middle subcell in the $\mathrm{UMM}^{\mathrm{B}}$ is now affected by the water absorption peak around $900 \mathrm{~nm}$. This region is within the bottom subcell range in the case of LM-Ge technology. In Figure 5 right, the EQE of the 4J solar cell is shown, where the EQE of the LM-Ge has been split in two. For the case of the $4 \mathrm{~J}$ solar cell, three SMRs will be considered $S M R_{\mathrm{sc} 2}^{\mathrm{sc} 1}, S M R_{\mathrm{sc} 3}^{\mathrm{sc} 2} S M R_{\mathrm{sc} 4}^{\mathrm{sc} 3}$ and compared to the $S M R_{\text {mid }}^{\text {top }}$ and $S M R_{\text {bot }}^{\text {mid }}$ of the primary LM-Ge technology. 

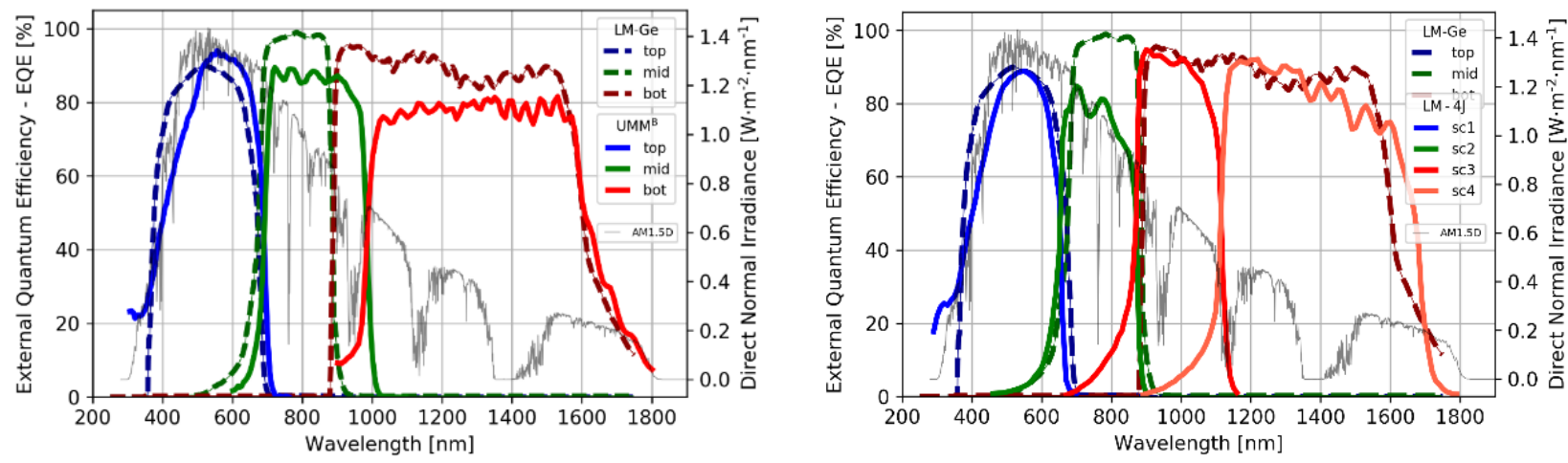

Figure 5. External Quantum efficiencies (EQE) of secondary technologies compared to primary Lattice-Matched Germanium based (LMGe). (left) Upright Metamorphic type $b\left(\mathrm{UMM}^{B}\right)$ [21] and (right) 4 junction solar cells based on wafer bonding (4J) [22]. Superimposed the AM1.5D G173-03 reference spectrum can be observed.

The green dots in Figure 6 and Figure 7 show the correlation for the direct comparison, that is, the $S M R_{\text {mid }}^{\text {top }}$ for secondary technologies (UMM ${ }^{\mathrm{B}}$ and $4 \mathrm{~J}$ ) are directly compared to the SMRs values of the primary technology (LM-Ge). Let's start with $\mathrm{UMM}^{\mathrm{B}}$ shown in Figure 6. The high dispersion found for the direct comparison is mainly caused by the differences in the EQE at the middle-bottom frontier, at around $900 \mathrm{~nm}$. This affects the correlation of both technologies and increases the $C V_{\text {RMSE }}$ to $2.36 \%$ for $S M R_{\text {mid. }}^{\text {top }}$. For the $S M R_{b o t}^{\text {mid }}$, since the middle subcell of $U_{M M}{ }^{B}$ technology sees the first water peak of absorption that sees the bottom subcell of LM-Ge, the impact is even stronger (high bias and $\left.\mathrm{CV}_{\mathrm{RMSE}}=3.22 \%\right)$.

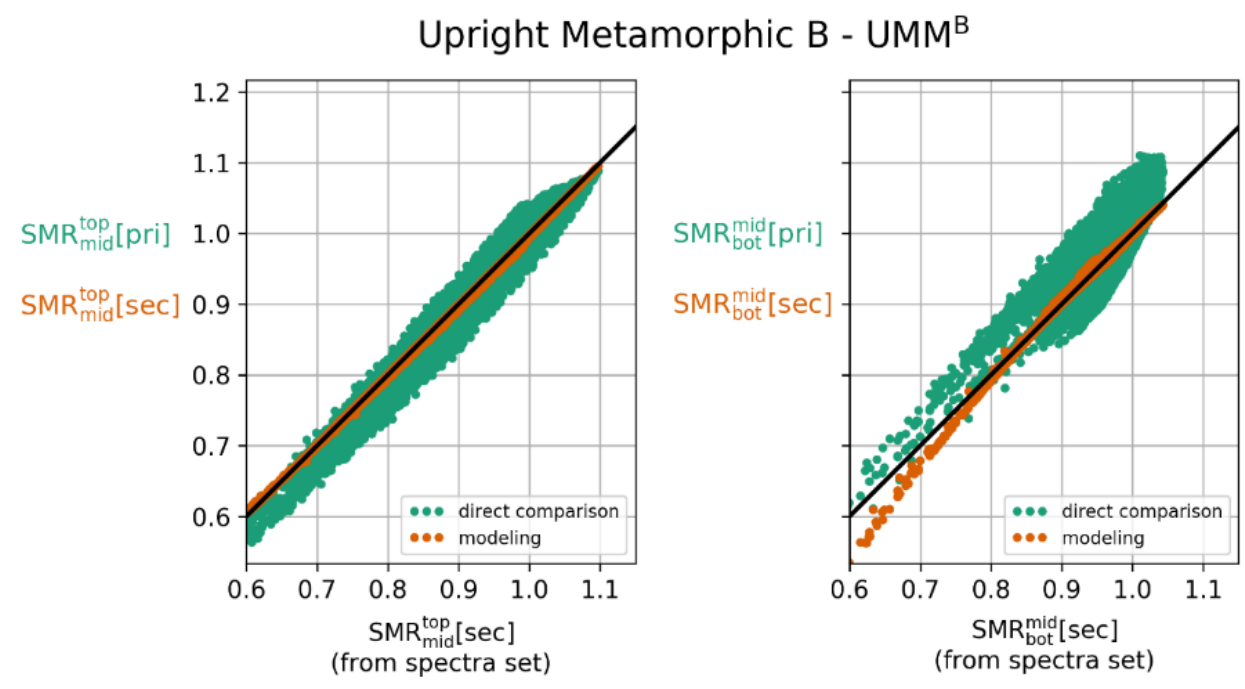

Figure 6. Correlation of $S M R_{\text {mid }}^{\text {top }}$ and $S M R_{b o t}^{\text {mid }}$ of $U \mathrm{UM}^{\mathrm{B}}$ technology (secondary) calculated assuming LM-Ge as primary. For both graphs, $x$-axes represent the SMRs for UMM ${ }^{B}$ technology calculated from the spectra data set. The $y$-axes represent the SMRs for LM-Ge technology (direct comparison) as well as the SMRs for the $U_{M M} M^{B}$ technology derived from the effective direct normal irradiances of LM-Ge using eq. (4) to (6) (modeling). The spectral data set has been obtained by means of SMARTS and using as input information annual data (year 2014) from the Madrid AERONET station. 

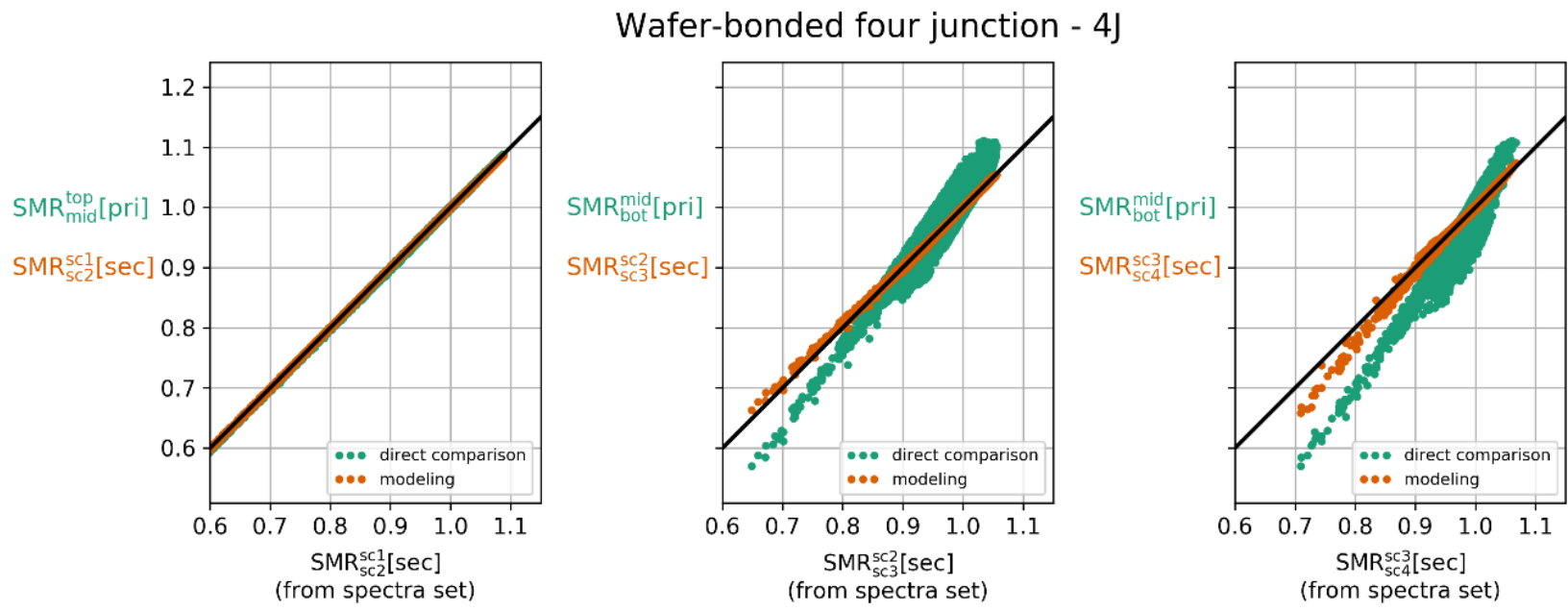

Figure 7. Correlation of $S M R_{s c 2}^{s c 1}, S M R_{s c 3}^{s c 2}$, and $S M R_{s c 4}^{s c 3}$ of $4 \mathrm{~J}$ technology (secondary) calculated assuming LM-Ge as primary. For both graphs, x-axes represent the SMRs for $4 \mathrm{~J}$ technology calculated from the spectra data set. The y-axes represent the SMRs for LM-Ge technology (direct comparison) as well as the SMRs for the 4J technology derived from the effective direct normal irradiances of LM-Ge using eq. (4) to (6) (modeling). The spectral data set has been obtained by means of SMARTS and using as input information annual data (year 2014) from the Madrid AERONET station.

Orange dots on Figure 6 show the correlation when the SMRs for $U_{M M}{ }^{B}$ are estimated based on the effective direct normal irradiance of the LM-Ge technology. In this case, the dispersion decreases and the $\mathrm{CV}_{\text {RMSE }}$ is significantly reduced to $0.34 \%$ and $0.50 \%$ for the $S M R_{\text {mid }}^{\text {top }}$ and $S M R_{\text {bot }}^{\text {mid }}$ repectively.

The results for the $4 \mathrm{~J}$ solar cell, depicted in Figure 7, show a very good correlation when directly comparing the SMRs involving the upper two junctions. Their similar EQEs lead to $C V_{R M S E}=0.16 \%$ when comparing $S M R_{\text {mid }}^{\text {top }}$ and $S M R_{\text {sc2 }}^{\text {sc1 }}$ (green dots in Figure 7). When using the model $S M R_{\mathrm{sc2}}^{\mathrm{sc} 1}$, a slight improvement is found as the $C V_{R M S E}$ reduces to $0.10 \%$. On the contrary, strong differences appear between the $S M R_{\mathrm{sc} 3}^{\mathrm{sc} 2}$ and $S M R_{\mathrm{sc} 4}^{\mathrm{sc} 3}$ when directly compared (green dots) to the LM-Ge $S M R_{\text {bot }}^{\text {mid }}$, with $C V_{R M S E}$ values of $2.18 \%$ and $2.88 \%$ respectively. Again, if $S M R_{\mathrm{sc} 3}^{\mathrm{sc} 2}$ and $S M R_{\mathrm{sc} 4}^{\mathrm{sc} 3}$ are modeled based on the effective direct normal irradiances of the $\mathrm{LM}-\mathrm{Ge}$, the error is reduced to $0.19 \%$ and $0.38 \%$, proving that the primary triple junction LM-Ge can also model the analyzed $4 \mathrm{~J}$ solar cell.

\section{Conclusions}

The definition of spectral indices with the capability of defining and constraining the solar spectral condition is essential for PV applications highly influenced by spectral variations, such as CPV using MJ cells. The three effective direct normal irradiance values obtained by means of the components (isotypes) of a 3J-Ge cell technology were proposed as a set of spectral indices. They are useful to evaluate the spectral impacts on MJ solar cell not only of the same technology but also of different technologies.
Based on this approach, correlations between the spectral indices of MJ cell technologies with different spectral responses were obtained for Madrid. SMR values were calculated through the effective direct normal irradiance values given by a set of components cells for a reference $3 \mathrm{~J}$ cell technology ( $3 \mathrm{~J} \mathrm{LM-Ge} \mathrm{in} \mathrm{our}$ case study). Then, based on those reference effective direct normal irradiance values, SMR values for alternative solar cell architectures have been estimated. This method was proven to achieve very good correlation even for cell solar whose EQE significantly differs from that of the reference LM-Ge, such as upright 3J metamorphic solar cells or wafer-bonded 4-junction solar cells. Furthermore, it was shown that in the case of reference (primary) and modeled (secondary) solar cell technology with similar EQE, it may be sufficient to use the SMR values obtained directly from the reference component cells. This approach is expected to work well for the same site or sites with similar spectral variation ranges.

\section{Acknowledgements}

We thank the AERONET network and in particular the principal investigators and their staff for establishing and maintaining the sites used in this investigation. This work has been supported by the Comunidad de Madrid through the program MADRID-PV-CM (S2013/MAE2780) and the Ministerio de Economía y Competitividad through the project ISCOGEN (RTC-2015-3982-3). Rubén Núñez is thankful to the Spanish Ministerio de Economía y Competitividad for his FPI grant. This work has received funding from the European Union's Horizon 2020 research and innovation programme within the project CPVMatch under grant agreement No 640873. 
The authors are solely responsible for the content of this work and it only reflects the author's view. The European Commission or any of their agencies are not responsible for any use that may be made of the information it

\section{References}

[1] C. A. Gueymard, "Parameterized transmittance model for direct beam and circumsolar spectral irradiance," Sol. Energy, vol. 71, no. 5, pp. 325-346, Nov. 2001.

[2] N. L. A. Chan, H. E. Brindley, and N. J. Ekins-Daukes, "Impact of individual atmospheric parameters on CPV system power, energy yield and cost of energy," Prog. Photovolt. Res. Appl., vol. 22, no. 10, pp. 1080-1095, Oct. 2014.

[3] R. Núñez et al., "Spectral study and classification of worldwide locations considering several multijunction solar cell technologies," Prog. Photovolt. Res. Appl., vol. 24, no. 9, pp. 1214-1228, Sep. 2016.

[4] R. Núñez, I. Antón, S. Askins, and G. Sala, "Atmospheric parameters, spectral indexes and their relation to CPV spectral performance," in AIP Conference Proceedings, 2014, vol. 1616, pp. 290-293.

[5] M. Theristis, E. F. Fernandez, F. Almonacid, and P. Perez-Higueras, "Spectral Corrections Based on Air Mass, Aerosol Optical Depth, and Precipitable Water for CPV Performance Modeling," IEEE J. Photovolt., vol. 6, no. 6, pp. 1598-1604, Nov. 2016.

[6] P. M. Rodrigo, E. F. Fernández, M. Theristis, and F. A. Cruz, "Characterization of the Spectral Matching Ratio and the Z-Parameter From Atmospheric Variables for CPV Spectral Evaluation," IEEE J. Photovolt., vol. 7, no. 6, pp. 1802-1809, Nov. 2017.

[7] P. M. Rodrigo, E. F. Fernández, F. M. Almonacid, and P. J. Pérez-Higueras, "Quantification of the spectral coupling of atmosphere and photovoltaic system performance: Indexes, methods and impact on energy harvesting," Sol. Energy Mater. Sol. Cells, vol. 163, pp. 73-90, Apr. 2017.

[8] G. Peharz, G. Siefer, and A. W. Bett, "A simple method for quantifying spectral impacts on multi-junction solar cells," Sol. Energy, vol. 83, no. 9, pp. 1588-1598, Sep. 2009.

[9] C. Domínguez, S. Askins, I. Antón, and G. Sala, "Characterization of five CPV module technologies with the Helios 3198 Solar Simulator," in 34th IEEE Photovoltaic Specialists Conference, Philadelphia, USA, 2009, pp. 1004-1008.

[10] C. Domínguez, I. Antón, G. Sala, and S. Askins, "Current-matching estimation for multijunction cells within a CPV module by means of component cells," Prog. Photovolt. Res. Appl., vol. 21, no. 7, pp. 14781488, Nov. 2013.

[11] R. Núñez et al., "Determination of spectral variations by means of component cells useful for CPV rating and design," Prog. Photovolt. Res. Appl., vol. 24, no. 5, pp. 663-679, May 2016.

[12] P. A. Hubanks, M. D. King, S. Platnick, and R. Pincus, "MODIS atmosphere L3 gridded product algorithm theoretical basis document," NASA Goddard Space Flight Cent., Greenbelt, MD, 2008.

[13] C. Gueymard, "SMARTS2: a simple model of the atmospheric radiative transfer of sunshine: algorithms and performance assessment," Florida Solar Energy Center Cocoa, FL, 1995. contains. This work made use of the Scipy stack [26], an open-source Python-based scientific computing environment.

[14] B. N. Holben et al., "AERONET-A federated instrument network and data archive for aerosol characterization," Remote Sens. Environ., vol. 66, no. 1 , pp. 1-16, 1998.

[15] A. Committee G03, "Tables for Reference Solar Spectral Irradiances: Direct Normal and Hemispherical on 37 Tilted Surface," ASTM International, t, e w, 2012.

[16] R. W. Mueller et al., "Rethinking satellite-based solar irradiance modelling: The SOLIS clear-sky module," Remote Sens. Environ., vol. 91, no. 2, pp. 160-174, May 2004.

[17] G. Siefer et al., "SOPHIA CPV module round robin: Power rating at CSOC," in AIP Conference Proceedings, 2014, vol. 1616, pp. 167-172.

[18] M. Victoria et al., "Tuning the current ratio of a CPV system to maximize the energy harvesting in a particular location," in AIP Conference Proceedings, 2013, vol. 1556, p. 156.

[19] I. Antón et al., "Power rating of CPV systems based on spectrally corrected DNI," AIP Conf. Proc., vol. 1477, no. 1, p. 331, 2012.

[20] D. Fuhrmann et al., "About 42\%-class CPV cells and paths beyond," presented at the CPV-9, Miyazaki (Japan), 2013.

[21] H. Nagai et al., "Development of the new DFK CPV module by NGCPV Japan-EU collaboration," in Proceedings of 6th World Conference on Photovoltaic Energy Conversion, Kyoto, Japan, 2014.

[22] F. Dimroth et al., "Wafer bonded four-junction GalnP/GaAs//GalnAsP/GalnAs concentrator solar cells with 44.7\% efficiency," Prog. Photovolt. Res. Appl., vol. 22, no. 3, pp. 277-282, Mar. 2014.

[23] Jaus, J., "Spectral Measurements Using Component Cells: Examinations on Measurement Precision," in 26th European Photovoltaic Solar Energy Conference and Exhibition, EU PVSEC. Proceedings, 2011, pp. 176181.

[24] R. Núñez et al., "Spectral network based on component cells under the SOPHIA European project," in AIP Conference Proceedings, 2015, vol. 1679, p. 030006

[25] R. Núñez, C. Jin, I. Antón, and G. Sala, "Spectral classification of worldwide locations using SMR indexes," in AIP Conference Proceedings, 2016, vol. 1766, p. 090007.

[26] F. Pérez, B. E. Granger, and J. D. Hunter, "Python: An Ecosystem for Scientific Computing," Comput. Sci. Eng., vol. 13, no. 2, pp. 13-21, Mar. 2011.

\section{Annex}

Coefficient for the model of the effective direct normal irradiances of a secondary MJ solar cell technology as a function of the effective direct normal irradiances of a primary technology, as described by eq. (4) to (6). In all the cases, the LM-Ge has been considered as the primary technology.

\begin{tabular}{|c|c|c|c|c|}
\hline & pri=top & pri=mid & pri=bot & $\mathrm{k}$ \\
\hline UMM $^{\mathrm{A}}$ & & & & \\
\hline Ctop_pri & 0.952610 & 0.058201 & -0.011833 & -0.335550 \\
\hline
\end{tabular}




\begin{tabular}{|c|c|c|c|c|}
\hline Cmid_pri & -0.023348 & 0.918845 & 0.128837 & -21.700184 \\
\hline Cbot_pri & -0.007204 & 0.030767 & 0.94483 & 28.366000 \\
\hline \multicolumn{5}{|l|}{$\mathrm{UMM}^{\mathrm{B}}$} \\
\hline Ctop_pri & 0.903418 & 0.113502 & -0.018619 & -0.387010 \\
\hline Cmid_pri & -0.014756 & 0.698751 & 0.388779 & -64.442748 \\
\hline Cbot_pri & -0.025013 & 0.119179 & 0.821978 & 74.758665 \\
\hline \multicolumn{5}{|l|}{$4 \mathrm{~J}$} \\
\hline Csc1_pri & 1.056885 & -0.071913 & 0.013763 & 1.271904 \\
\hline Csc2_pri & 0.064248 & 0.955365 & -0.013770 & -5.164690 \\
\hline Csc3_pri & -0.121919 & 0.575338 & 0.569855 & -21.418561 \\
\hline Csc4_pri & 0.051699 & -0.190059 & 1.066351 & 64.763328 \\
\hline
\end{tabular}

